\title{
Foreshortening produces errors in the perception of angles pictured as on the ground
}

\author{
Marta Wnuczko $^{1} \cdot$ Karan Singh $^{1} \cdot$ John M. Kennedy ${ }^{1}$
}

Published online: 5 November 2015

(C) The Psychonomic Society, Inc. 2015

\begin{abstract}
Observers viewed pictures of a simulated ground plane and judged the orientation of lines pictured as lying on the ground. We presented three lines at a time and manipulated three factors: (1) the declination of the lines below the horizon (depicting distance to the target angles), (2) their length, and (3) whether or not they converged to a point on the horizon. Only the first factor had a substantial effect on these errors. We conclude that perspective foreshortening in pictures produces errors in perceived 3-D orientation. Our explanation is based on the different rates of change of elevation and azimuth with distance.
\end{abstract}

Keywords 3-D perception · Depth and shape perception $\cdot 2-\mathrm{D}$ shape and form $\cdot$ Space perception

Painted on highways, arrows often indicate a lane on which we can drive straight ahead. From a distance, the arrowhead may look highly obtuse, say $150^{\circ}$. As we drive closer, the arrowhead seems to shrink, and as we come close we may see that the angle is only $10^{\circ}$ or so. Here we will examine this phenomenon and show how it relates to linear perspective.

Evident to some extent in pictures from antiquity (Keuls, 1978; Pinnell, 1996), linear perspective was first described explicitly in the Renaissance (Alberti, 1435-1436/1966; Killian, 2012; Kubovy, 1986). In linear perspective, if an object recedes into the distance, the angle it subtends in the observer's optic array gets smaller. Also, if a circle tilts, increasing its optic slant, it subtends slimmer ellipses. As a

John M. Kennedy

kennedy@utsc.utoronto.ca

1 University of Toronto, 1265 Military Trail, Toronto, Ontario M1C1A4, Canada result, perspective "foreshortening" can result from two effects, and compression in pictures often results from the combined result of visual angle shrinkage with distance and an increase in optical slant with distance along the ground. Because of foreshortening, a linear-perspective picture can show parallel lines on the ground by means of lines that converge toward a single point on the horizon, as the bold obliques do in Fig. 1.

In Fig. 1, the 3-D "scene" features are projected as rather different 2-D "screen" features. The task of perception is to use optic array and "screen" features to detect "scene" features. Each of the bold obliques makes a unique screen angle with the vertical, but they all depict the same angle with a $z$ line in the scene. Does vision take the unique screen angles as the scene angles? Almost, but not quite, we propose, for pictures like Fig. 1. The visual system fails to take foreshortening into account adequately, and in particular, we suggest that it makes little use of the screen feature "convergence to a point on the horizon," which could have been highly informative, in principle.

Information in perspective assists the perception of 3-D scenes (Banks, Cooper, \& Piazza, 2014; Bruggeman, Yonas, \& Konczak, 2007; Durgin, 2014; Hammad, 2015; Juricevic \& Kennedy, 2006; Sedgwick, 1986; Todorović, 2005; White, 1956; Williams \& Durgin, 2015). However, for centuries scholars have argued about how (from Alhazen, 1039/1989, and Berkeley, 1709/1975, to moderns such as Kubovy, 1986; Yang \& Purves, 2003; and Kennedy \& Wnuczko, 2015). We focus here on the perception of scene angles, and our suggestion is that foreshortening leads to major errors.

Durgin (2014) noted that $z$ distances are often underestimated visually. For a standing observer, distances from the vantage point, such as $20 \mathrm{~m}$ along a $z$-line, may be estimated as about $14 \mathrm{~m}$; that is, "near-walking" $z$ distances are underestimated visually by about $30 \%$. A meter stick 


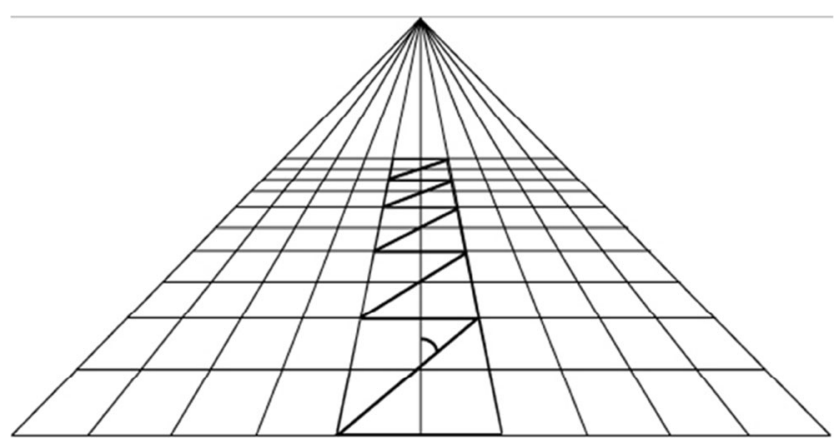

Fig. 1 Trapezoids projected by square tiles. Bold oblique lines depict diagonals in a path of tiles receding into the distance. The vertical line in the middle depicts a $z$-line in the 3-D scene- a line on the ground from below the vantage point, stretching to the horizon. The arc marks an angle on the picture surface depicting a target angle on the ground. The lines depicting the sides of the path converge. On the picture surface, the bold obliques form angles with the middle vertical of about $50^{\circ}, 60^{\circ}, 65^{\circ}, 70^{\circ}$, and $75^{\circ}$, reading upward from the arc, but the diagonals in the scene are always at $45^{\circ}$ to the $z$-line. The reader's impression may be that the lowest $\left(50^{\circ}\right)$ bold line depicts a diagonal of a square in the scene, but the other scene angles depicted by the bold lines are larger than $45^{\circ}$ and increase in size toward the horizon. Also, the obliques converge, though in the scene the diagonals are parallel

lying on the ground on a $z$-line, at a 10-m distance, would be judged to be about $70 \mathrm{~cm}$ long. Exocentric distances between spots on a ground are underestimated, say Ooi, $\mathrm{Wu}$, and $\mathrm{He}$ (2001; see also Ooi \& He, 2007; Wu, He, \& Ooi, 2007; Wu, Ooi, \& He, 2004). They suggest that the error is due in part to a bias toward seeing a ground plane as a concave curve. It may also be due to issues regarding vision's imperfect use of angular declination (Durgin, 2014; Rand, Tarampi, CreemRegehr, \& Thompson, 2012; Williams \& Durgin, 2015). More generally, the shrinkage of an apparent ground plain may also be due to visual problems with objects slanted to the line of sight. Schmidt, Khan, Kurtenbach, and Singh (2009) tested observers with picture of a cylinder, lying on its side, with its top near the observer's vantage point. The task was to indicate the middle of the cylinder. The middle was judged to be nearer the observer than was true, meaning that the farther-away part of the cylinder was apparently shrunk in comparison to the near part. Furthermore, and of key importance for our account, Loomis, Da Silva, Fujita, and Fukushima (1992) reported that visual estimates of $z$ distances are reduced in comparison to impressions of similar horizontal extents that are at right angles to the $z$ line. That is, if the meter stick at $10 \mathrm{~m}$ egocentric distance is turned $90^{\circ}$, lying on the ground, it will look larger. Likewise, Durgin considered that a vertical stick $1 \mathrm{~m}$ in height, with a base $10 \mathrm{~m}$ distant from the observer, is judged to have a height of about $1 / 7$ th the egocentric distance, not 1/10th. In Durgin's terms, visual scaling of distance differs from the scaling of height and width (Wagner, 1986). Specifically, Durgin argued that $z$ distances appear reduced in comparison to $y$-verticals and $x$-horizontals. Here, we examine the implications of this reduction. Following Gibson
(1950), we relate it to perspective projections from (1) depths ( $z$ distances) and (2) widths ( $x$-horizontals, or "azimuths"). We suggest that vision does not track ratios between the two correctly. Predictions follow for the perception of angles.

By way of an introduction to foreshortening in pictures, consider the diagonals shown in Fig. 1. The higher the bold lines are in "elevation" in the picture, the more they tilt toward the horizontal. Acute angles, such as the one marked by an arc, made with a vertical line in the middle of the picture increase toward $90^{\circ}$. Consider the angles they represent on the ground to be "target angles." In the scene, they are the angles on the far side of an intersection. Since parallels define them, they are all equal. On the screen, they are on the upper side of an intersection, and they are unequal.

Foreshortening increases upward in Fig. 1 along with several other factors. The smaller the declination of an intersection from the horizon, the farther the target angle. Simultaneously, the farther the target angle on the ground, the larger the optic slant at the intersection. (Optic slant is the angle between the line from the vantage point and the vertical from the ground at the target.) However, a screenangle increase - with elevation on the 2-D picture surface, with distance, and with optic slant - does not indicate that the target angles in the 3-D scene are changing size. They are all $45^{\circ}$, since they all belong to the diagonals of squares. But they do not look it. Is vision simply using the screen angles?

The acute angles increase with height in the picture because of different projections from their "elevations" and "azimuths" (Gibson, 1950; Wnuczko, 2015). On the 2-D picture surface, $z$ distances in the 3-D scene project vertical "elevations." Distances at right angles to the $z$ distances are $x$ distances in the 3-D scene, and project as horizontal "azimuths" ("widths," in Durgin's terms).

A square tile in the central path in the 3-D scene always extends one unit in $z$ distance and one unit in $x$ distance, but its projection on the picture surface changes with distance. Of interest for foreshortening, on the 2-D picture surface, projections from the square's unit-sized $z$ distances (vertical "elevations" on the screen) shrink quickly — at a quadratic rate with distance. As distance increases, the $x$-distance projections (i.e., azimuths or "widths) shrink slowly — only at a linear rate. The difference produces many of foreshortening's features. As distance increases, square tiles in the path project slimmer and slimmer trapezoids in the picture. The more distant the tile, the more its azimuth (horizontal) projection differs from its elevation (vertical) projection. Naturally, the slimmer the trapezoid, the more its diagonal approaches horizontal. In other words, as a square tile approaches the horizon, foreshortening makes its diagonals project closer to horizontal in the picture.

Ideally, visual judgments of target angles should take account of proximity to the horizon in the picture (Durgin, 2014; Rand, Tarampi, Creem-Regehr, \& Thompson, 2011). Along 
the $z$-line, declination from the horizon plus the screen angle specifies the scene angle. But if vision does not account for how rapidly elevation foreshortens in comparison to azimuth, errors will increase with distance. Indeed, if no account at all is taken of foreshortening, visual judgments would equal the screen angles.

To understand that convergence could be useful to vision, consider viewing Fig. 1 from its correct vantage point, the center of projection. Let the distance from the correct vantage point to a picture's central vanishing point be $1 \mathrm{~m}$. The lines depicting the diagonals of the central path of square tiles would converge to a point on the horizon (called the "distance point") $1 \mathrm{~m}$ from the vanishing point (Kubovy, 1986). If perception uses this geometric fact, it could compare a little-foreshortened angle, one nearby in 3-D and low in the picture, and then adopt that size for the other, more distant and more foreshortened angles.

Furthermore, if the lines converge to a point on the horizon line farther than $1 \mathrm{~m}$ from the central vanishing point, then the 3-D scene's diagonals belong to wide rectangles, ones with their long axis in the azimuth dimension ("horizontal" in the picture). Correspondingly, if they converge to a point on the horizon closer than $1 \mathrm{~m}$ from the central vanishing point, the 3-D scene's diagonals belong to long rectangles (short axis in the azimuth dimension). Vision could tell that in the 3-D scene, the target acute angle in the first case is $45^{\circ}$, greater than $45^{\circ}$ in the second, and smaller in the third. Does vision make use of these facts? If they are parallel to the picture surface, parallels project as parallels. If they lie in the ground plane (or a plane parallel to a ground plane), their point of convergence lies on the horizon, at key distances from the vanishing point. (If the parallel lines do not lie in the ground plane, or in another plane that is parallel to the ground, their projections also converge to a single point, but that point does not lie on the ground plane's horizon.)

To test vision's handling of foreshortening, we used pictures showing three obliques on a textured ground, one with parallels, like Fig. 2A and B. Target angles depicted as far off are in the most-foreshortened parts of the scene and may have large errors. Figure 2A's lines converge to a single point on the horizon to show parallels in the scene. It might support relatively small errors for the distant obliques, since parallels all make the same angles with a $z$-line, and the nearby line might be seen relatively accurately. In contrast, Fig. 2B's three white lines offer three different intersections and might not allow vision to minimize errors for distant obliques. Alternative possibilities are that perception is simply governed by the screen angles at the intersections, or, importantly, that percepts lie partway between the screen angle and scene angle, partially taking account of foreshortening.

Shorter lines than those in Fig. 2A and B, ones not reaching the horizon, do not provide explicit convergence to a point on the horizon. Short lines thus could allow greater error. Alternatively, target angles might be judged simply using
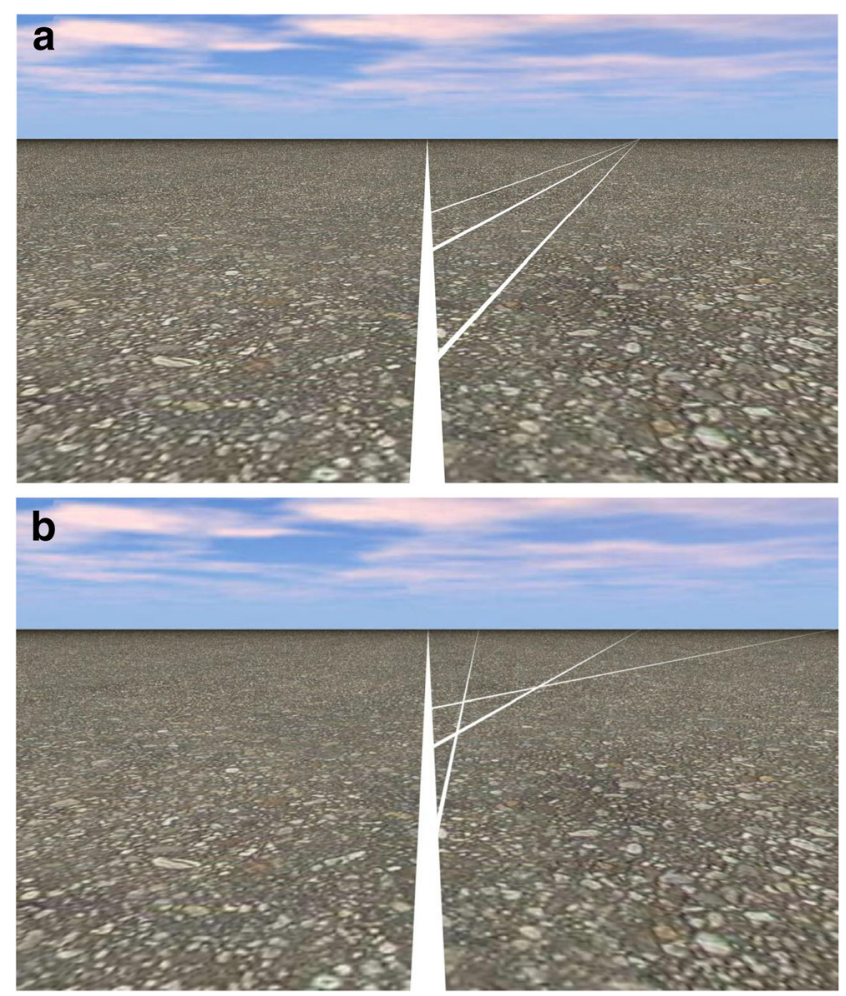

Fig. 2 Perspective pictures of obliques. Picture A depicts three parallel obliques on the ground, at near, middle, and far distances at $20^{\circ}$ to the $z$ line on the ground. The acute angles made by the white lines depicting the obliques with the vertical on the picture surface are approximately $42^{\circ}$, $62^{\circ}$, and $72^{\circ}$, reading from the base of the picture upward. Also reading from the base of the picture upward and from near to far in the scene, B depicts obliques intersecting the $z$-line at $5^{\circ}, 20^{\circ}$, and $35^{\circ}$ on the ground. In $\mathrm{B}$, the $35^{\circ}$ oblique on the ground projects a white line in the picture that intersects the horizon line close to the side edge of the picture. To show a $45^{\circ}$ oblique's intersection with the horizon line, the picture would have to be wider. In B the acute angles on the picture surface are approximately $12^{\circ}, 62^{\circ}$, and $79^{\circ}$, reading from the base of the picture upward. In both pictures, the projection is from oblique lines intersecting the $z$-line at 1.5 , 3.0 , and $4.5 \mathrm{~m}$ from a point on the ground below the viewing eye of an observer sitting $1.0 \mathrm{~m}$ in front of the picture. (Pictures programmed by Bruno de Araujo and Ricardo Jota.)

information in features near the target angle at the intersection of the oblique and the $z$-line, along with the declination of the intersection with respect to the horizon.

In short, we asked about the visual errors related to foreshortening, and whether explicit convergence to a single point on the horizon has much of an impact in practice.

\section{The present experiment}

Observers estimated target acute angles in linear perspective pictures of obliques on an asphalt ground. The displays depicted three parallel obliques simultaneously (Fig. 2A) or three nonparallel obliques simultaneously (Fig. 2B). Foreshortening increases with the $z$ distance along the ground (and optic slant and decreasing declination in the picture from 
the horizon). Increased foreshortening might induce larger errors, despite information about parallels.

\section{Method}

\section{Participants}

Ten adults (six women, four men; mean age $=27$ years, $S D=$ 4.9) participated. All were right-handed and reported normal or corrected-to-normal vision. The participants were recruited by e-mail from the University of Toronto Dynamics Graphics Project participant pool. Eight of the participants were current students, two were alumni (one female and one male, who graduated in 2009 and 2014, respectively). Each student had a major in either arts or sciences, and each student had a different major. Participants were given $\$ 20$ for participation.

\section{Apparatus}

The pictures were projected onto a large framed screen about three times the width of the image and twice the height. The projections measured $0.77 \mathrm{~m}$ in height and $1.39 \mathrm{~m}$ in width, and subtended about $40^{\circ} \times 62^{\circ}$ at a distance of $1 \mathrm{~m}$ from a vantage point on the normal from the vanishing point. The pictures, devised for a vantage point $1.22 \mathrm{~m}$ above the lab floor and $1 \mathrm{~m}$ from the screen, were created by a custom program using the OpenGL graphic API, running on Microsoft Windows, and written as part of the University of Toronto Dynamics Graphic Project.

Like Fig. 2A and B, the pictures depicted a textured ground plane extending to a horizon. The texture depicted small stochastically regular closed-contour forms on a gray "asphaltlike" pavement. In the pictures, the density of the forms increased from the base of the display to the horizon. The texture gradient on the picture surface was a linear-perspective projection of a ground plane at the vantage point of the observers. A $z$-line was projected as a vertical white line, diminishing in width, extending from the base of the picture to the depicted horizon. Three obliques were projected as white lines at varying angles to the $z$-line. They also became narrower with distance.

The $5^{\circ}$ oblique projected at screen angles of $12^{\circ}$ (near), $25^{\circ}$, and $33^{\circ}$ (far). The $20^{\circ}$ projected at $42^{\circ}, 62^{\circ}$, and $72^{\circ}$ screen angles, and the $35^{\circ}$ oblique projected at $60^{\circ}, 75^{\circ}$, and $79^{\circ}$ screen angles. The $z$-line met the horizon line at $90^{\circ}$. Naturally, the angle that an oblique makes on the screen with the horizon line is given by $90^{\circ}$ minus the oblique's target screen angle. Also, on the screen, the vertical distance down the $z$-line from the horizon line to the intersection with the oblique, taken together with the target's screen angle, can specify a horizontal distance. In particular, on the screen they specify the distance from the central vanishing point to the horizon's intersection with the oblique. The picture surface was vertical and parallel to the frontal plane of the observer.

The distances from the point on the ground below the vantage point to the intersection of an oblique with the $z$-line on the ground depicted behind the picture surface were 1.5, 3.0, and $4.5 \mathrm{~m}$ for the near, middle, and far obliques, respectively. The distances from the horizon line to the intersections of the lines depicting obliques with the vertical line depicting the $z$ line were $0.13,0.4$, and $0.6 \mathrm{~m}$, with declinations from the horizon of $7.4^{\circ}, 21.8^{\circ}$, and $31^{\circ}$, respectively. (With respect to the observer's vantage point, the optic slant of the screen at the intersections is given by the declination.) The $35^{\circ}$ oblique intersected the horizon line $0.65 \mathrm{~m}$ from the central vanishing point.

The lengths of the obliques varied. On any given trial, the picture showed either three short, three medium, or three long obliques: They were depicted as short $(0.5 \mathrm{~m})$, medium $(2 \mathrm{~m})$, or long (fully extended, reaching the horizon) on the 3-D scene's ground.

Two sets of pictures were presented, and every picture showed three parallel or three nonparallel obliques. Each observer judged displays with short, medium, and long obliques. The angles on the depicted ground plane between the obliques and the $z$-line varied. In the "parallel" condition, the picture depicted obliques at $5^{\circ}$ or $20^{\circ}$ or $35^{\circ}$ to the $z$-line on the ground. In the "nonparallel" condition, observers were shown each of the three possible angles $\left(5^{\circ}, 20^{\circ}\right.$, and $\left.35^{\circ}\right)$ on a given trial. That is, each possible angle was represented by an oblique. For example, in one condition, the near oblique was presented at $5^{\circ}$ to the $z$-line, the middle at $20^{\circ}$, and the far at $35^{\circ}$. All six combinations of near-middle-far and $5^{\circ}-20^{\circ}-35^{\circ}$ were tested. In the parallel condition, all three obliques were presented to the observer at a given angle $\left(5^{\circ}\right.$ or $20^{\circ}$ or $\left.35^{\circ}\right)$ to the $z$-line. Thus, in total there were 3 distances $\times 3$ angles $\times 3$ lengths, making 27 conditions for each observer. (Adopting a set of angles determined whether or not the condition had parallel lines.)

The angles were estimated by observers using a device made from a circular analog clock. The numbers on the face of the clock were painted over with white paint and covered with a protractor. The long minute hand was fixed to the 12:00 position and was used in reference to the direction of the $z$ line. The short hour hand was adjustable. The acute angle between the minute hand and the hour hand represented the target angle being observed.

\section{Procedure}

Observers sat $1 \mathrm{~m}$ from the screen, viewing monocularly by wearing glasses with one eyepiece opaque, with their viewing eye about $1.2 \mathrm{~m}$ above the ground. The height of the chair was adjusted to bring the viewing eye to the level of the horizon in the picture. The response device (the clock) was held by 
observers on their lap. The room was windowless, but room lighting was on and the response device was plainly visible.

The target angle was pointed out to the observer. The instructions directed the observer to estimate the acute angle on the ground shown in the picture as if it lay behind the screen. Observers were asked to judge a target angle on the ground, not the angle on the screen. After a practice trial, the $27 \mathrm{ex}$ perimental displays were presented in a random order. In each trial, observers were asked to estimate each of the three target angles. Six question orders were randomized across trials.

Half of the observers used their left eye, and half their right. Between trials, observers were asked to close their eyes and sit with a straight back. The distance to the screen and eye height above the floor were measured to ensure that the vantage point was approximately maintained, and then the observers were asked to look at the target angle and to report their estimate of its size.

\section{Results}

Figure 3 shows the differences between the reported angle and the scene angle (overestimations), differences between the screen and reported angles, and differences between screen and scene angles. Table 1 summarizes the reported angles. Consider a brief description of the overestimations and then a statistical analysis.

Supporting the hypothesis that foreshortening increases apparent angles, the actual mean of the scene angles was $20^{\circ}$, but the mean of the reported angles were very much larger, $46.5^{\circ}$. As predicted, increases in foreshortening led to larger errors (the mean reported angle for near target angles was $40.7^{\circ}$, for the mid-distance was $46.7^{\circ}$, and for the far distance was $52.1^{\circ}$, a total increase of $11.4^{\circ}$ ).

Naturally, the reported angles also increased with target angle. The mean reported angle for the $5^{\circ}$ target angle was $26.6^{\circ}$, for the $20^{\circ}$ target angle it was $50.3^{\circ}$, and for the $35^{\circ}$ target angle it was $62.7^{\circ}$.

Observers did not make much use of convergence to a single point on the horizon, for the mean reported angle for parallel obliques was $45.4^{\circ}$, only slightly less than for obliques that were not parallel $\left(47.7^{\circ}\right)$. Information at intersections may have been what was important, since the reported angles were no different for longer obliques.

Now we consider statistical significance. The reported angles were converted to errors, with positive meaning overestimation, and analyzed with a 2 (parallel [single point of convergence] vs. nonparallel) $\times 3$ (near, middle, or far distance on the ground to the oblique $) \times 3\left(5^{\circ}, 20^{\circ}\right.$, or $35^{\circ}$ target angle $) \times 3$ (short, medium, or long length) repeated measures analysis of variance. In brief, errors increased with distance, the parallel condition had slightly smaller errors, and the length of the obliques had little or no effect.

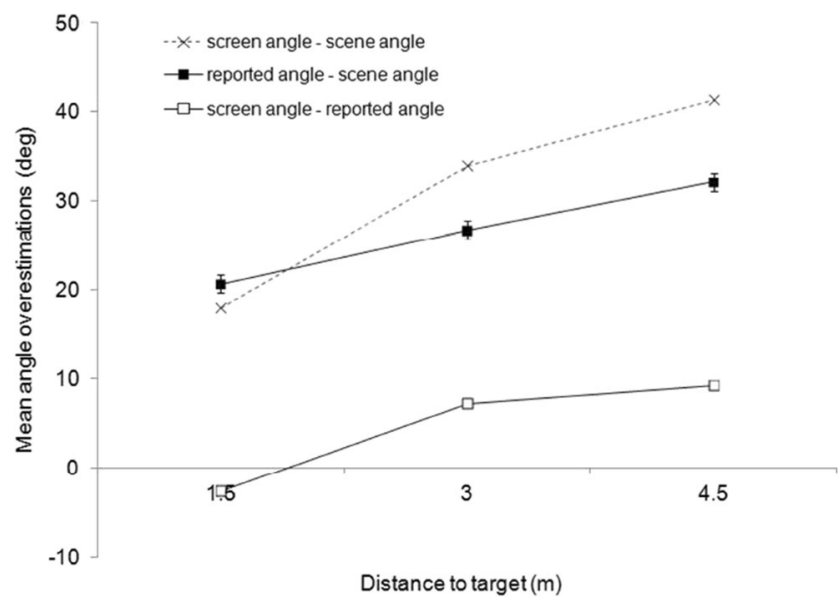

Fig. 3 Mean overestimations of scene angles as a function of distance to the target, as well as differences between the screen angle and the reported angle and between the screen angle and scene angle

The target angles were overestimated by means of $20.7^{\circ}$ $(S D=3.6), 26.7^{\circ}(S D=3.9)$, and $32.1^{\circ}(S D=4.7)$ at the near, middle, and far distances, respectively. The errors increased with distance, and hence foreshortening, $F(2,18)=47.67, p<$ $.001, \eta^{2}=.9$. Angles were overestimated only slightly more $\left(2.3^{\circ}\right.$, less than $\left.10 \%\right)$ in the nonparallel condition $\left(M=27.7^{\circ}\right.$, $S D=5.5)$ than in the parallel condition $\left(M=25.4^{\circ}, S D=5.6\right)$, $F(1,9)=20.4, p=.001, \eta^{2}=.7$. Errors did not vary with the lengths of the obliques, $F(2,18)=1.14, p=.3, \eta^{2}=.1$, but errors did increase with target angle, $F(2,18)=19.8, p<.001$, $\eta^{2}=.7$. The interactions were all nonsignificant $(p \mathrm{~s}>.05)$.

\section{Discussion}

Target angles were overestimated, and they were overestimated more as distance and foreshortening increased.

Table 1 In degrees, the target angles, screen angles, and mean reported angles in the parallel and nonparallel conditions for three distances (near, mid, and far)

\begin{tabular}{lll}
\hline $\begin{array}{l}\text { Target Angle } \\
\text { (Near, Mid, Far) }\end{array}$ & $\begin{array}{l}\text { Screen Angle } \\
\text { (Near, Mid, Far) }\end{array}$ & $\begin{array}{l}\text { Mean Reported Angle } \\
\text { (Near, Mid, Far) }\end{array}$ \\
\hline Parallel & & \\
$5,5,5$ & $12,25,33$ & $21,25,31$ \\
$20,20,20$ & $42,62,72$ & $44,49,55$ \\
$35,35,35$ & $60,75,79$ & $56,60,67$ \\
Nonparallel & & \\
$5,20,35$ & $12,62,79$ & $21,51,70$ \\
$20,35,5$ & $42,75,33$ & $45,65,33$ \\
$35,5,20$ & $60,25,72$ & $58,28,58$ \\
$5,35,20$ & $12,75,72$ & $21,67,58$ \\
$20,5,35$ & $42,25,79$ & $45,30,69$ \\
$35,20,5$ & $60,62,33$ & $57,52,32$ \\
\hline
\end{tabular}


The mean overestimation was considerable, over $200 \%$, and the reports were close to the screen angle, though less so for the farthest angles (more than $95 \%$ of the variation in the reported angles can be accounted for by variation in screen angle, with $r=.98$ ). Correlation does not indicate mean size, of course, so it is important to add that the target angles had a mean size in the scene of $20^{\circ}$, but they were judged to have, on average, a size of $40.7^{\circ}$ at the near distance and a size of $52.1^{\circ}$ at the far distance. Even small target angles nearby were overestimated considerably; $5^{\circ}$ angles were reported as more than $20^{\circ}$. Vision failed to follow the linear versus square compressions due to distance. Change in distance should produce increasing error, we argued, and, indeed, error did increase by $11.4^{\circ}$. Furthermore, information about parallels that was given by a common convergence point on the horizon reduced errors surprisingly modestly, at best. Indeed, no observer gave the same angle for all distances in any parallel condition. Thus, having information available does not mean that it will be used.

The overestimation was least at $1.5 \mathrm{~m}$. The nearest object was the least foreshortened, and in principle it could have been accurate and used to anchor the perception of more foreshortened angles (Wu, Ooi, \& He, 2004; Hammad et al., 2008), but in practice it revealed little if anything about the other targets in the parallel condition.

The range of the target angles tested here, $5^{\circ}$ to $35^{\circ}$, is useful for two reasons. First, errors can be large: $5^{\circ}$ can be reported with an $85^{\circ}$ error, given that the ceiling is $90^{\circ}$. In contrast, target angles of $45^{\circ}$ can only approach $90^{\circ}$, and so have a maximum error of $45^{\circ}$, and target angles of $75^{\circ}$ have a maximum error of only $15^{\circ}$. Only testing with small scene angles offers the opportunity for large errors. If we had given target angles of $1^{\circ}$ or less, viewed at larger distances than the ones used here, the percent overestimation could have been as large as desired. Second, the $35^{\circ}$ scene-angle oblique at the far distance projected a screen angle of $79^{\circ}$, in theory not much short of the ceiling effects at $90^{\circ}$.

Were observers reporting screen instead of scene angles (Hammad, 2015; Holway \& Boring, 1953)? This question deserves careful consideration. The two larger target angles $\left(20^{\circ}\right.$ and $\left.33^{\circ}\right)$ at the two larger distances were reported as being smaller than the screen angles, and therefore showed a very modest amount of constancy (about 0.2 in terms of Thouless ratios, in which 0 is no constancy-the screen value - and 1 is perfect constancy - the scene value). The nearby $5^{\circ}$ target angles, on the other hand, were reported as larger than the screen angles. Other target angles were reported close to the screen angles. Why might this be? We will argue here that in pictures, the "screen angle" projected from a target angle on the ground, such as a $45^{\circ}$ angle, ranges all the way from $0^{\circ}$ (forelengthened) to $90^{\circ}$ (foreshortened), and in theory the reported angle should only vary from correct to $90^{\circ}$.
Certainly, screen angles provide information about the scene angles, via the optic array at the vantage point. However, both the mid- and far-distance screen angles here were significantly larger than the reported angles [mid-distance, $t(9)=4.5, p<.01$; far distance, $t(9)=5.4, p<.001]$. Also, the near-distance screen angles were numerically smaller than the reported angles, though at best marginally significantly different $[t(9)=1.8, p=.1]$. Why might this be?

There are two extreme conditions for the screen angleclose to the horizon, and almost underfoot. When an angle is close to the horizon, it is squashed vertically, providing a screen angle of about $90^{\circ}$. In this case, all scene angles should be reported as essentially $90^{\circ}$. For this condition, the realworld observation with which we began is relevant. Highway arrows showing "straight-ahead" look highly obtuse from afar, at a large optic slant. Close up, the arrowhead is found to be acute, perhaps $10^{\circ}$.

In contrast, consider the pictorial situation with a square that is almost underfoot. Let a screen extend vertically down to the ground, in contact with the near edge of the square. This screen subtends a wide angle, close to $90^{\circ}$ downward from the normal stretching horizontally from the vantage point to the screen. Wide-angle screens subtend more than $45^{\circ}$ from this normal. They are the condition for forelengthening (Kennedy \& Juricevic, 2002) or "marginal distortions" (Kubovy, 1986), shown in Fig. 4.

In this case, the screen projection from the $z$-dimension of the square is longer than the one from its azimuth. In the limit, forelengthening of the $z$-dimension of the square approaches infinity, so the screen angle projected from a target angle would approach $0^{\circ}$. However, the scene angle should be reported correctly-a $45^{\circ}$ target angle, for example, should be reported as about $45^{\circ}$ - showing good perceptual constancy. The reason is that the scene angle is portrayed on a surface almost underfoot - that is, almost directly below the observer. The normal from the vantage point down to the depicted ground, and close to vertical, meets the target at close to $90^{\circ}$, and optic slant is minimal.

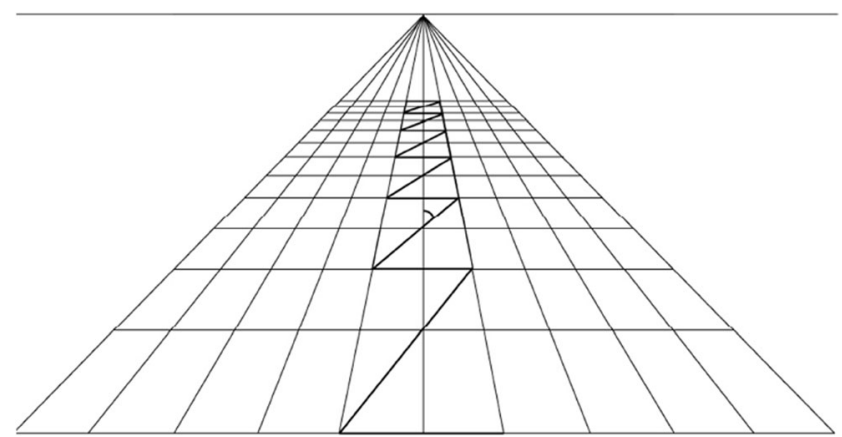

Fig. 4 Picture of square tiles on the ground. The upper obliques are inside foreshortened forms, as in Fig. 1, in which the azimuth projection is larger than the vertical one. The lowest oblique, however, is in a "forelengthened" shape, in which the vertical projection is larger than the azimuth 
This observation condition is as helpful as viewing a target on a wall at head height, for optic slant is minimized. The target angle would thus be reported correctly, to within a few degrees (Hammad, Kennedy, Juricevic, \& Rajani, 2008).

Between the extremes of highly forelengthened and foreshortened, what occurs to reported angles? Forelengthened, the screen angle should be smaller than the reported angle. Screen angles would then expand as forelengthening decreases and gives way to foreshortening. For much of foreshortening, if vision takes partial account of its effects, the screen angle should be larger than the reported angle. But foreshortening reaches $90^{\circ}$ as a limit, at which point the reported angle catches up and screen angles equal the reported angles, and constancy is lost.

In principle, viewing targets increasing in distance while lying on the ground is just another way to vary the optic slant of a target. For ecological surfaces, distance and optic slant are distal variables that affect a proximal variable - the rate of change of the optic-texture density in the observer's optic array. Declination from the horizon is also in the optic array, confounded with texture density, and, in pictures, is specified by obliques at particular screen angles intersecting the horizon line at particular distances, as we noted in the Apparatus section above. Our effects cannot be ascribed unambiguously to one or the other feature; disentangling them will be a task for the future. Given the confound, results the same as ours could be obtained using pictures of obliques on tilted textured surfaces at a fixed distance from the observer (Sedgwick \& Levy, 1985).

We stress that the observers were tested with a picture, not a real scene. Our apparatus was not immersive, and observers viewed monocularly, without physical head restraints. In these circumstances, screen surfaces are visible, and pictures "flatten" the perceived scene relative to the real scene (as Sedgwick, 1986, in particular has emphasized; see also Koenderink \& van Doorn, 2003). The more evident is the picture surface's texture, the greater the flattening. The effects of foreshortening in the real world, implied by a .7 underestimation of near walking distance (Durgin, 2014), may be larger for pictures. That is, the underestimation of $z$ distance might be .7 in a real scene but, say, .6, even less, in a picture of the scene. But both picture perception and ordinary, direct perception should both contain errors due to foreshortening.

The texture elements in Fig. 2 have elevation and azimuth components. Consider the number of texture units crossed by a line (Hammad, 2015; Knill, 2003): Observers sensitive to these could detect angles (Durgin, 2014; Holway \& Boring, 1953; Juricevic \& Kennedy, 2006; Saunders \& Backus, 2006). They might pick a point on the oblique, and then, as if examining a graph, by reading horizontally they might notice the number of texture units in the azimuth from the point to the $z$ - line, say three. That is, reading horizontally from the point, they might count three texture units. Continuing to read the optic input as if it were a graph, they might then count the elevation units corresponding to the three azimuth units. That is, they might read vertically down the $z$-line to the level of the intersection, counting texture units alongside the $z$-line. If this number is also three, then the line intersects the $z$-line at $45^{\circ}$. The ratio of azimuth to elevation units is information about the target angle. If texture units near the intersections help observers make angle estimations in this fashion, it is little wonder that varying the lengths of the obliques had no significant effect and that a common convergence point on the horizon, far from the intersection, was only of modest use. That is, if observers evaluate features near intersections (Todd, Thaler, \& Dijkstra, 2005), extra length is not needed.

Of interest, the standard deviations decreased slightly, numerically, as the lengths of the obliques increased in the nonparallel condition (near $S D=4.4$, middle $S D=3.8$, far $S D=3.5$ ), but not in the parallel condition (near $S D=5.9$, middle $S D=6.7$, far $S D=5.6$ ). Also, the $S D$ s were numerically larger in the parallel condition. It may be that the parallel condition leads observers to use more kinds of information-convergence in addition to texture and elevation - which increases accuracy slightly but, since more kinds of information are being consulted, fosters increased variability at the same time. However, it cannot be that observers use these sources of information effectively and correctly, or their results would have been flawless. As Koenderink and van Doorn (2003, p. 272) have said, "what enters the brain need not enter the mind." Rather, besides excellent optical information, theory needs a source of perceptual error. Our prime suggestion about angles is that vision is deeply influenced by foreshortening, but it underestimates the rate of change in elevation versus azimuth.

If a depicted scene is tiled uniformly, such as by $1-\mathrm{m}$ squares, the optical evidence for the target angles might be much more evident than is the case for stochastically regular textures. Indeed, late Renaissance Italian pictures, after the discovery of linear perspective, frequently depicted floors with square tiles. Of interest, the present result may even help explain why the discovery of linear perspective came late in the 50,000-year history of picture making. The parallel obliques in our figures look as if they hit the $z$-lines at different angles, and this would have impeded artists from discovering what specifies parallels!

In conclusion, tested with linear-perspective pictures, vision fell into error as angles foreshortened and elevations shrank faster than azimuths. Despite its key information for constancy of angle perception, convergence to a single point on the horizon reduced errors only slightly. Perspective foreshortening introduces errors, even if its use is necessary for the perception of space. 
Author note We thank our reviewers, notably Hal Sedgwick, for many helpful comments.

\section{References}

Alberti, L. B. (1966). On painting (J. R. Spencer, Ed.). New Haven, CT: Yale University Press. (Original work published 1435-1436).

Alhazen, I. (1989). Book of optics. In A. I. Sabra (Ed., \& Trans.), The optics of Ibn-Haytham (Vol. 1, pp. 1-367 ). London, UK: University of London, Warburg Institute. (Original work published 1039).

Banks, M. S., Cooper, E. A., \& Piazza, E. A. (2014). Camera focal length and the perception of pictures. Ecological Psychology, 26, 30-46. doi:10.1080/10407413.2014.877284

Berkeley, G. (1709/1975). Philosophical works; including the works on vision. M. Ayers (ed.). London: Dent.

Bruggeman, H., Yonas, A., \& Konczak, J. (2007). The processing of linear perspective and binocular information for action and perception. Neuropsychologia, 45, 1420-1426. doi:10.1016/j. neuropsychologia.2006.11.004

Durgin, F. H. (2014). Angular scale expansion theory and the misperception of egocentric distance in locomotor space. Psychology and Neuroscience, 7, 253-260. doi:10.3922/j.psns.2014.032

Gibson, J. J. (1950). The perception of the visual world. Boston, MA: Houghton Mifflin.

Hammad, S. (2015). Components of optical information for depth induce a picture-surface angle illusion. Unpublished doctoral dissertation, University of Toronto, Toronto, Canada.

Hammad, S., Kennedy, J. M., Juricevic, I., \& Rajani, S. (2008). Angle illusion on a picture's surface. Spatial Vision, 21, 451-462. doi:10. $1163 / 156856808784532554$

Holway, A. G., \& Boring, E. G. (1953). A methodological study of sizedistance perception. Journal of Psychology, 35, 143-153.

Juricevic, I., \& Kennedy, J. M. (2006). Looking at perspective pictures from too far, too close, and just right. Journal of Experimental Psychology: General, 135, 448-461. doi:10.1037/0096-3445.135.3.448

Kennedy, J. M., \& Juricevic, I. (2002). Last but not least: Foreshortening gives way to forelengthening. Perception, 31, 893-894.

Kennedy, J. M., \& Wnuczko, M. (2015). What is a surface? In the real world? And pictures? In P. F. Bundgaard \& F. Stjernfelt (Eds.), Investigations into the phenomenology and the ontology of the work of art (pp. 89-107). Heidelberg, Germany: Springer.

Keuls, E. C. (1978). Plato and Greek painting. Leiden, The Netherlands: Brill.

Killian, J. (2012). That deceptive line: Plato, linear perspective, visual perception, and tragedy. Journal of Aesthetic Education, 46, 89-99.

Knill, D. C. (2003). Mixture models and the probabilistic structure of depth cues. Vision Research, 43, 831-854.

Koenderink, J. J., \& van Doorn, A. J. (2003). Pictorial space. In H. Hecht, R. Schwartz, \& M. Atherton (Eds.), Looking into pictures: An interdisciplinary approach to pictorial space (pp. 239-299). Cambridge, MA: MIT Press.

Kubovy, M. (1986). The psychology of perspective and Renaissance art. Cambridge, UK: Cambridge University Press.

Loomis, J. M., da Silva, J. A., Fujita, N., \& Fukusima, S. S. (1992). Visual space perception and visually directed action. Journal of Experimental Psychology: Human Perception and Performance, 18, 906-921. doi:10.1037/0096-1523.18.4.906
Ooi, T. L., \& He, Z. J. (2007). A distance judgment function based on space perception mechanisms - Revisiting Gilinsky's equation. Psychological Review, 144, 41-454. doi:10.1037/0033-295X.114. 2.441

Ooi, T. L., Wu, B., \& He, Z. J. (2001). Distance determined by the angular declination below the horizon. Nature, 414, 197-200. doi:10.1038/ 35102562

Pinnell, W. H. (1996). Perspective rendering for the theatre. Carbondale, IL: Southern Illinois University Press.

Rand, K. M., Tarampi, M. R., Creem-Regehr, S. H., \& Thompson, W. B. (2011). The importance of a visual horizon for distance judgments under severely degraded vision. Perception, 40, 143-154. doi:10. $1068 / \mathrm{p} 6843$

Rand, K. M., Tarampi, M. R., Creem-Regehr, S. H., \& Thompson, W. B. (2012). The influence of object ground contact on perception of distance and size under severely degraded vision. Seeing and Perceiving, 5, 425-447.

Saunders, J. A., \& Backus, B. T. (2006). Perception of surface slant from oriented textures. Journal of Vision, 6(9), 882-897. doi:10.1167/6.9.

Schmidt, R., Khan, A., Kurtenbach, G., \& Singh, K. (2009). On expert performance on 3-D curve drawing tasks. In C. Grimm \& J. J. LaViola (Eds.), Proceedings of the EUROGRAPHICS Symposium on Sketch-based Modeling (pp. 133-140). New York, NY: Association for Computing Machinery.

Sedgwick, H. A. (1986). Space perception. In K. R. Boff, L. Kaufman, \& J. P. Thomas (Eds.), Handbook of perception and human performance (Vol. 1, 21:1-21:57). New York, NY: Wiley.

Sedgwick, H. A., \& Levy, S. (1985). Environment-centered and viewercentered perception of surface orientation. Computer Vision Graphics and Image Processing, 31, 248-260.

Todd, J. T., Thaler, L., \& Dijkstra, T. M. H. (2005). The effects of field of view on the perception of 3D slant from texture. Vision Research, 45, 1501-1517. doi:10.1016/j.visres.2005.01.003

Todorović, D. (2005). Geometric and perceptual effects of the location of the observer vantage point for linear-perspective images. Perception, 34, 521-544. doi:10.1068/p5225

Wagner, M. (1986). The metric of visual space. Perception \& Psychophysics, 38, 483-495.

White, J. (1956). Perspective in ancient drawing and painting. London, UK: Society for the promotion of Hellenic studies.

Williams, M. J. C., \& Durgin, F. H. (2015). Direct manipulation of perceived angular declination affects perceived size and distance: A replication and extension of Wallach and O'Leary (1982). Attention, Perception, \& Psychophysics, 77, 1371-1378. doi:10. 3758/s13414-015-0864-y

Wnuczko, M. (2015). Foreshortening leads to overestimation of angles. Unpublished doctoral dissertation, University of Toronto, Toronto, Canada.

Wu, B., He, Z. J., \& Ooi, T. L. (2007). The linear perspective information in ground surface representation and distance judgment. Perception \& Psychophysics, 69, 654-672. doi: 10.3758/BF03193769

Wu, B., Ooi, T. L., \& He, Z. J. (2004). Perceiving distance accurately by a directional process of integrating ground information. Nature, 428 , 73-77. doi:10.1038/nature02350

Yang, Z., \& Purves, D. (2003). A statistical explanation of visual space. Nature Neuroscience, 6, 632-640. 